Empowerment: through the smoke and past the mirrors?

\author{
Kevin Morrell \\ and \\ Adrian Wilkinson \\ Loughborough University Business School \\ Ashby Road \\ Loughborough \\ Leicester \\ LE11 3TU
}

Tel: 01509228273

Fax: 01509223960

Email: a.j.wilkinson@lboro.ac.uk 


\begin{abstract}
This paper offers a five-fold typology for empowerment, which is intended to make it easier to assess and develop empowerment initiatives in a climate where much rhetoric surrounds the term. A real-world organizational sketch for each type follows an initial theoretical description and aphorism. The implications for HRD practitioners of each type of empowerment are also outlined. No exclusive or definitional treatment is offered for what we feel to be an elastic term. Instead, the framework is held up as a potential catalyst for provoking ideas about empowerment, or grouping existing ideas on how to empower. We sympathise with those who feel the term is redundant because it has been subsumed by the 'developmental rhetoric' (Clutterbuck 1998), and recognise that such rhetoric can be a barrier to change and learning (Harrison 1997). Nonetheless, we argue that instead of abandoning the term empowerment altogether, it can be beneficial to see what has been accomplished under the existing banner.
\end{abstract}

\title{
Introduction
}

Suppose someone offered you a magic spell which could

- increase the performance standards of your staff

- give you more time at work to set strategy and plan developments

- improve the service you give to your customers

And then told you the cost: nothing at all! Would you turn the offer down? I doubt it... This 'magic spell' is called empowerment (Mitchell Stewart 1994: vi).

There are a number of ways of trying to make sense of the term 'empowerment'. This paper offers a brief summary of some of them, and then proposes a typology. Part of the motivation for writing this paper has been to contribute to the current debate, where it has been recognised that much rhetoric surrounds the term 'empowerment' (Argyris 1998; Clutterbuck 1998), and that this rhetoric can be a barrier to both organizational and individual learning (Harrison 1997: 8-9). We suggest that the scope for particular developmental or performance enhancing initiatives need not be limited by the recognition of some general underlying rhetoric. It is less important to seek to invent a new term for empowerment activity such as 'mutuality of benefit' (Clutterbuck 1998), than to understand what different things may be being done under the existing banner. 
By outlining five types of empowerment, and giving examples of each type in practice, we hope this can make it easier to see beyond the smoke and mirrors surrounding this term. The article does not give the recipe for a magic spell, but may offer a guide to appraising existing 'empowerment initiatives', and developing future ones. Much of the literature on empowerment offers great promises to employers seeking 'to empower' (Byman 1991; Mitchell Stewart 1994). Other writers are more sceptical (Adler 1993). A former tutor of one of the authors refuses even to say the word empowerment because they believe that whenever it is used this is invariably the sign of bad or downright immoral management practice! The authors do not subscribe to either of these extreme views. We are unable to offer employers and managers an 'Open Sesame', and unwilling to try to exorcise the spirit of something which may improve the employment relationship and employment practice. Instead what we offer is a pragmatic reappraisal of the term, intended to provide some focus amid the hocus pocus!

\section{New Lamps for Old?}

The term empowerment has been widely associated with the popular management movements of Total Quality Management (TQM) and Human Resource Management (HRM). We can also see the promises empowerment offers in terms of the 'excellence' movement, where the customer is 'king'. In this sense, empowerment should enable organizations to be more immediately responsive to their customers, as decision-making is devolved. In the mainstream HRD literature, there is a similar sense of devolution of power, for example where employees have responsibility for, and ownership of their learning (Megginson, Joy-Matthews and Banfield 1993: 149). Yet the roots of empowerment can be traced back further than the contemporary TQM and HRM/D movements (Wilkinson 1997). We could look at empowerment in terms of it being an 'answer' to the excesses of scientific management. Ostensibly, it offers workers the chance to decide how they want to work, rather than leaving that to the people who are 'paid to do the thinking'. This interpretation does not so much make an economic case for empowerment, as a moral or human relations case. Here, empowerment could be seen in a similar light to the Quality of Working Life (QWL) movement in the 1970's. 
Alternatively we could see it as a new name for a much older phenomenon where, given certain industrial conditions, productivity and quality of work are enhanced when workers have control and responsibility over work processes. A good example of this might be pre industrial revolution cottage industries, where craftsmen had responsibility for production. The idea that workers should have control over work processes has once again resurfaced, as change in technology for many industries has made traditional managerial control mechanisms redundant. The modern 'craftsman's' task has again become too complicated to measure, or supervise. Nowadays however, instead of technology disenfranchising workers (as it did with many pre industrial revolution craftsmen), it can actually be seen as a liberating force.

\section{Smoke and Mirrors}

Yet it need not be the case that empowerment is driven by progressive changes in the context. The effects of recession, and widespread redundancy may have meant that organizational survivors simply have more work to do, and the (cynical) label for this is that they have become empowered. Developing this, we can see how empowerment can become a weasel word, framed in smoke, aggrandized by mirrors. The 'smoke' of empowerment may hide the fact that there is no real increase in or reconstitution of workers' power, instead empowerment proves to be a more insidious mechanism for control. Traditional control systems (supervisors) may be replaced by more sophisticated measuring systems or management may monitor team performance so that peer pressure acts as a controlling force. In addition, the implicit end result of successful introduction of empowerment is culture change, which can be seen as another form of control. The 'mirrors' of empowerment may exaggerate the benefits to employees of increased responsibility or 'ownership'; increased responsibility which does not bring increased reward, and ownership which applies to the process but not the profits. Instead empowerment becomes a slick piece of re-labelling designed to get more for less.

The term is complex and subject to differing interpretations. The implications of this are that it will not be perceived in the same way by different organizations, nor will people 
within the same organization think of empowerment in the same way. HRD practitioners may identify with how such rhetoric can prejudice the introduction of change. We can readily recognize the undercurrent of cynicism surrounding the term empowerment, but it is worthwhile remembering that vogue terms, such as 'learning organization' within development may be viewed with similar suspicion, and this can in turn prohibit learning. Importantly too, even if we overcome cynicism, the meaning of such terms may be expressed in equally vague or overly optimistic terms. It is not difficult to see the parallels between use of the term empowerment, and Garvin's (1993) portrayal of learning organizations;

...discussions of learning organizations have often been reverential and utopian, filled with near mystical terminology. Paradise, they would have you believe is just around the corner... Sound idyllic? Absolutely. Desirable? Without question. But does it provide a framework for action? Hardly.

Perhaps even more significantly, critical analysis of what key themes within the writing on empowerment mean in practice can inform how these concepts are used within HRD. For example, the notion that 'ownership' can be scaled, or continuous rather than binary may resolve one paradox HRD practitioners face daily, as follows. The chance of someone learning effectively is enhanced if they have 'ownership' both during the learning process, and when applying that learning. Yet the agenda and scope of formal development within organizations is by and large not owned by employees. Equally, the business case for HRD relies on establishing a link between development and the bottom line, which the employee again doesn't own. Reconciling this obvious tension is far more difficult if we have a simplistic either-or construction of ownership.

\section{What's the Magic Word?}

That empowerment is a management buzzword means attempts to explicitly introduce 'empowerment' will be affected by people's preconceptions. Whether these are favourable or unfavourable can affect the success of these initiatives. Even favourable expectations can prove problematic if the initiatives then fail to live up to these. Also, 
not every industry sector, or organization will form an equally receptive context for empowerment. Different organizations could respond to the same initiatives very differently. Response will be influenced by (among other things); commitment and integrity of senior management, the organization's history and culture, and the nature of work carried out by that organization. Much of the writing on empowerment does not look at the term in any context or offer an adequate definition. Instead what empowerment actually means is left rather vague, and the term is used very loosely. Whilst we are unwilling to give a concrete definition for something which is not a unified term, empowerment at its simplest would commensensically be associated with the redistribution of power. In business terms however, it is often taken to mean some form of employee involvement, designed by management to generate commitment and enhance employee contribution, in contrast to its wider meaning in other literatures.

It is worth remembering that because employers decide whether and how to empower employees, it may be less of a redistribution of power, and more like a reconstitution of it. Empowerment is also often portrayed as a universal solution, appropriate to all sectors, all organizations and all employees. Consequently, its advocates may trivialise or ignore conflict within organizations, assuming employees will universally welcome the chance to 'release their trapped potential'. Instead, it is likely that in any organization, whether initiatives gain the commitment of employees will be contingent. In fact, it would be rather ironic if there were one way of achieving empowerment, given that any coherent empowerment philosophy should allow some choice for all. Presumably, this choice should extend to how empowerment is introduced?

\section{A Typology}

Empowerment schemes can be of many different forms, which in turn may reflect a variety of diverse management motivations. They can range from the mechanistic (involving structural change) to the more organic (concerned with attitudes / culture). Notwithstanding this note of caution, we can identify five main types of scheme, namely information sharing, upward problem solving, task autonomy, attitudinal shaping and 
self-management (Wilkinson 1997). One could argue that what you call something is less important than the results you get by doing it, but as we have discussed, the established sense of a term can act as a barrier to change and / or learning. It is worth reflecting on how we might usefully distinguish between "empowerment initiatives" (in name only) and initiatives which empower ('the real deal'). One hoped for advantage of the five-fold classification is that it makes it easier to identify when and in what way something can be seen as empowering. It can also help the design of context-sensitive initiatives, as well as aid tracking of these initiatives via specific, measurable goals.

\section{Information sharing}

\section{Knowledge is the mother of all virtue; all vice proceeds from ignorance (Proverbs)}

We can analyse information sharing by looking at it in terms of the direction, i.e. downward, horizontal or upward. Where emphasis on 'cascading' information down via newsletters, team briefings etc. will have one type of impact on employees, emphasis on horizontal communication, potentially through the use of cross-functional teams and quality circles will have another effect. Reliance on instruments of employee 'voice' such as trade unions or more fashionably 'speak-up schemes' and suggestion schemes will have another impact. Each of these could be seen as empowerment initiatives.

Downward communication is a form of involvement, though it may be seen as pseudoparticipation (Pateman 1970), i.e. not 'you will do this', but 'this is why you will do this'. Nonetheless, if properly initiated it does mean management may be speaking directly to their employees, who may feel this is better than the sense of being given the 'mushroom treatment' i.e. kept in the dark etc. Improving horizontal communication can prove helpful in sharing expertise across different areas, promoting organizational learning and in achieving strategic goals. Encouraging upward communication gives us potentially the strongest example of empowering through communication. In this form of communication employees can be seen as actively informing management rather than being neutral (horizontal communication) or passive / receptive (downward communication). Yet there are problems too in how this is implemented. Unions may 
not prove receptive to ideas of empowerment, and still less to alternative (competing) channels of communication. Also, new forms of communication may not be well received by employees, and prove difficult to manage. Employees' perceptions of communication initiatives can be influenced both by preconceptions (where a scheme is being introduced) and by the ongoing management of a scheme. These perceptions will influence how information is received and acted upon, and thereby how successful any initiative is. For example, traditional institutions of employee voice (e.g. trade unions, grievance procedures) are vastly different in character from suggestion schemes, or speak-up schemes. Each of these latter, more modern versions of upward communication could be viewed cynically and with more suspicion by workers. In the case of speak-up schemes, getting the most from people may prove difficult if they feel insufficiently secure to voice dissatisfaction via, what is a management owned channel. In the case of suggestion schemes, managers may face a dilemma. Workers can feel they don't receive just reward for improvements that are adopted, yet disenfranchised if they feel their suggestions are ignored. As well as seeking to establish particular instruments and modes of communication, it is worth considering a more developmental possible end goal of this type of communication. The briefest and best definition of a learning company according to Pedler et al. is Revan's idea that organizations should encourage 'upward communication of doubt'. This neatly captures two aspects of this type of empowerment. Firstly and obviously, information can travel up the hierarchy. Secondly, and not so obviously, there needs to be the confidence to express uncertainty. 


\section{Sketch: Motorola University}

Motorola has gained attention in management literature owing to its establishing a 'university'. Based in Chicago, this 'university' has a campus, a range of excellent facilities, and several Deans, including a Dean of electronics and a Dean of quality.

As well as this centre for learning opportunities, individual sites have very powerful means of sharing information relating to training. The site at Cork has a dedicated multi-media centre, and its own library, which staff have access to.

This and other learning centres form powerful means of sharing information, and, we suggest, are evidence of empowerment. Significantly though, there is awareness within Motorola of the dangers of rhetoric. Motorola's training manager in Cork states, "When you talk about teamwork, it implies you haven't got it and saying empowerment implies a control culture." 


\section{Upward Problem Solving}

Ask an impertinent question and you are on the way to the pertinent answer (Bronowski)

Upward problem solving is similar in some ways to the dimension of information sharing, and there are again differing ways this kind of empowerment can be realised. Suggestion schemes may form a part of such upward problem solving, as may the ability of workers to identify problems and then inform management of them. Both these though are really a form of upward delegation. More significant impact can be made where workers (either individually or collectively) identify, and then solve problems that is when they are given the freedom not just to 'ask impertinent questions', but to provide the answers too. This can be seen as a special instance of Argyris and Schön's idea that organizational learning can be facilitated via 'organizational inquiry', which they define as;

...the intertwining of thought and action that proceeds from doubt to the resolution of doubt... doubt is construed as the experience of a "problematic situation," triggered by a mismatch between the expected results of action and the results actually achieved. (1978: 11)

We can also identify this type of empowerment with Morton's (1994) idea that we should see workers as having two jobs, one being to carry out specified tasks, and the other being to look for improvement. This fits well with the language of continuous improvement, 'kaizen' and other TQM tools and techniques. Perhaps the clearest example of this type of empowerment is within the philosophy of TQM, where teams can have final overall responsibility for a process or product.

A paradigm example of this kind of approach can be seen at Xerox, and their use of what they call 'X-Teams'. 
Sketch: Xerox and 'X-Teams'

Xerox has historically been associated with the development and implementation of leading edge quality initiatives. They were amongst the first wave of companies to adopt the language and techniques of Quality, forced into a drastic change in corporate philosophy largely owing to complacency and the expiry of many of their copyrights.

One of the more recent Quality initiatives at Xerox has been the development of their 'X-Teams'. These were set up to enable groups with different skills and backgrounds to create business process improvement.

Team members are chosen from different functions, and these teams operated with a great deal of discretion, reporting outside the established hierarchy. Though there is less emphasis on them now, as Xerox is undergoing significant restructure, these teams formed prime examples of how organizations can initiate improvement via upward problem solving.

The name 'X-Team' indicates the importance of a cross-functional aspect, which can help creativity and promote idea sharing, as people from traditionally different functions interact. The second important characteristic of these teams is the degree of autonomy they are allowed, and it is the combination of these two elements that allow for them to be effective. 


\section{Task autonomy}

Give us the tools and we will finish the job (Churchill)

It is perhaps easiest to see how task autonomy would operate if we look at it in a manufacturing context. Instead of assembly line workers being monitored by a supervisor, introduction of task autonomy could mean the removal of an inspection role, and restructuring of the work team, so the group as a whole has responsibility for (for example) quality control. Sometimes referred to as self-managing teams, these teams not only have responsibility for the basic work process, but are empowered in the stronger sense of having responsibility of traditional personnel / HR areas such as health and safety, and training and development. These teams can be seen as an extension or evolution of the idea of quality circles (Hicks R and Bone D 1990: 19). In their book on the learning company, Pedler et al. (1997) include 'empowered teams' as one of the 11 characteristics of a learning organization. In the sense of being empowered teams, selfmanaging teams are similar to the X-Teams at Xerox described above, but whereas XTeams would only meet intermittently, typically to look at a process outside their normal remit, members of self-managing teams work day-to-day alongside one another. 
Sketch: Yardley

Working in teams of about eleven, the success of teamworking at Yardley has led to improvements in working conditions, as well as bottom line benefits (Arkin 1995), leading to the company’s spending $£ 30,000$ to set up a meeting room for these teams. What is encouraging to those wishing to set up such teams, is the apparent ease with which groups formed, according to the business director;

The teamworking started in an almost ad hoc way, which didn't need any form of training or management involvement. All it needed was to get internal 'customers' and 'suppliers' to talk to each other. So initially we were not very keen to describe what we were doing as total quality or teamworking or empowerment, but to say this is common sense (ibid. p. 31).

It is interesting to note the initial reluctance to describe what was done as empowerment, but alongside this 'common sense', it is important to credit Yardley for establishing enabling structures, which have provided the necessary legitimacy and resources for these teams to manage themselves. After initial successes, the role of these teams became more formalised, and were supplemented by a range of management actions, including the establishment of a meeting room, and the setting up of an employee development programme which was facilitated by a new training centre and full time training co-ordinator. 


\section{Attitudinal shaping}

\section{A drop of honey catches more flies than a gallon of gall (Abraham Lincoln)}

Here, emphasis is on the psychological dimension of empowerment. It is perhaps easiest to see this in a service context, where employees' internalising of company values is seen as key to encouraging new behaviours. There may be no change in organizational or work structure, but employees can be educated / trained to 'feel empowered', which in turn means they are more confident interacting with customers. This may sound rather sinister, and superficial. It could even be that this approach is wrongheaded, and that bringing about change in how people feel about empowerment is something that can only be done after real change has taken place, as at Yardley. In other words, changed behaviour leads to changed attitudes, rather than the reverse (Beer et al 1990). The lack of any change in roles and responsibilities may mean that the only result from the customer's perspective is a better apology, rather than an improved service and such initiatives have been criticised as being merely 'smile campaigns'. But these criticisms may ignore the (possibly unpalatable) fact that a change in people's attitudes is often a desired result of those who hope empowerment can be their 'magic spell'. Part of the promise of creating an 'empowerment culture' is that people's attitudes will change. Nonetheless, it is important to realise that attitudinal shaping alone is unlikely to bring about real change. Neither is it likely that such changes in attitude will persist without supporting mechanisms and reorganization of the work itself. The importance which organizations attribute to attitudinal shaping is nonetheless notable, and may have resonance for HRD writers such as O'Donnell and Garvan, who identify the need for a wide ranging change first and foremost in the attitudes of people within organizations with respect to the relationship between training and industrial competitiveness (1997). 
Sketch: 3M

The Minnesota Manufacturing and Mining company (3M) has a reputation for innovation and creative problem solving, supported within a culture of entrepreneurship. Partly this is fostered by stories from the company's history, but also $3 \mathrm{M}$ has a powerful device for ensuring the character of entrepreneurship does not wither and die.

$3 \mathrm{M}$ uses what is referred to as the ' $15 \%$ rule' (Ghoshal and Bartlett 1998: 42), so called because this represents the amount of time available to certain employees to pursue their own 'bootleg projects'.

Admittedly, 3M has a powerful starting base, with a strong history of innovation, and it is fortunate to attract the pick of the labour market. They may not be so much shaping attitude, but selecting / attracting it. Even so, the example of the $15 \%$ rule shows how such devices can support a desired ethos of (here) entrepreneurialism. Their philosophy is memorably recounted in Ghoshal and Bartlett's book, thus:

At the foundation of everything in $3 \mathrm{M}$ is a deep, genuine and unshakeable belief in the ability of the individual. Surrounding it are a series of organizational policies and management practices that build on and leverage that basic belief (p. 43). 


\section{Self management}

This above all: to thine own self be true (Shakespeare)

This is perhaps the hardest form of empowerment to introduce into a work place. It is rare to see this type of empowerment in any real sense, although we may find evidence of it in particular industry contexts and professions. It is easy to see how barristers and surgeons can have great discretion over significant aspects of their work, yet it is perhaps harder to see how these professions can extend this discretion equally to others working alongside them. What self-management should mean ultimately, is that the division between managers and their employees is eroded. We can see some evidence of this as rhetoric in the way that many firms do not refer to their employees or workers, but to 'colleagues' or even 'partners' and 'associates'. Another example of an employment practice that erodes the division between management and employees is harmonisation. Employees at Nissan, for example wear the same uniform regardless of their formal 'rank'. These changes alone do not constitute true self-management. It may not be possible or desirable to extend this kind of autonomy to all levels of the workforce. Once again we must bear in mind that such a change in the employment practice will be context sensitive. It is easier to see this kind of initiative working in an advertising agency than at a hospital. Alternatively, some forms of employee ownership may encourage or lead to self-management. This may be particularly so where employees can be given greater control of their professional development, thereby creating greater opportunity for themselves. Some of the literature on careers and personal development emphasises this need and responsibility for employees to build employability or become 'intrapeneurs' (Baruch Y. and Peiperl M. 1997: 357) and presumably the desire for self-management is what motivates many to set up their own organizations. Although providing this degree of scope and freedom may be impossible for any organization, using this form of empowerment could help retain some expert employees. $3 \mathrm{M}$ 's ' $15 \%$ rule' may show the way to a compromise, and perhaps even more optimistically we might see that some employees may only have the opportunity for a significant level of freedom if they have the support (even patronage?) of an organization in the first place. 
Sketch: Semco

It is hard to talk meaningfully at length about empowerment without mentioning the names of Semco, and Ricardo Semler. What is perhaps most striking about management at Semco is their extension of the idea of teams with autonomous dimensions, to teams which are wholly self-managing.

Teams at Semco operate with a great deal of autonomy with responsibility over recruitment, development and managing their own budgets. In addition to this though, individual workers at Semco also have discretion over setting their own salary. This freedom is counterbalanced by the fact that whichever team wants to recruit them has to be prepared to pay for their services - out of their budget. It may be that the team structures at Semco show us a way to reconcile a central paradox within the rhetoric of empowerment. Control and autonomy can co-exist. 


\section{Conclusion}

The five types of empowerment shown here are not mutually exclusive. They can and do overlap. Some types will be more or less appropriate and realisable depending on the particular industrial or organizational context. Managing any significant change in the employment relationship is difficult. Often the writing on empowerment oversimplifies the nature of such change. Some of the key assumptions that lead to this oversimplification have been outlined here. Empowerment can take many forms and although it may not deliver all that is promised of it, it may benefit both employees and employers. That the extent of these benefits is likely to be limited does not mean they should be discounted totally. As Pfeffer (1994) suggests;

one should compare programmes not to some ideal, but to the situation that would exist in their absence. In other words, just because a programme doesn't solve every problem or move the organization all the way, particularly initially, to where it wants to be does not mean that it is a failure. A programme fails when it produces either no sustained change or else change that is dysfunctional and ineffective.

No firm or ideal definition of empowerment is offered here. Indeed the idea that there is 'one way' to define empowerment seems internally flawed. Even so, the framework here can offer a method for categorising and thereby assessing any 'empowering' initiative. Analysis of what lies behind the smoke and mirrors of empowerment can shed light on the rhetoric surrounding other terms in business and management. It is less helpful, we argue, to invent new terms and wholly abandon established ones, than to critically assess the meaning these established terms have in particular contexts. Equally however, it is important not be swayed by rhetoric, neither should we use such terms unthinkingly as they may mean different things to different people. These differences can be a barrier to communication and to learning. Discussion of the HRD implications of the typology offers a means of linking HRD to one aspect of HRM strategy. There are also implications for HRD practitioners in the analysis of themes of involvement and ownership, which find expression in the typology. 


\section{References}

Adler P. (1993) 'Time and Motion Regained', Harvard Business Review, Jan-Feb. Argyris C. (1998) 'Empowerment: The Emperor's New Clothes', Harvard Business Review, Jan-Feb.

Argyris C. and Schön D. A. (1978) Organizational Learning: a theory of action perspective, Addison-Wesley, Reading Mass.

Baruch Y. and Peiperl M. (1997) 'High-fliers: glorious past, gloomy present, any future?', Career Development International, 2 (7)

Beer M. Eisenstat R. and Spector B. (1990) 'Why Change Programmes Don’t Produce Change', Harvard Business Review, Nov-Dec.

Byman W. (1991) Zapp! The Lightning of Empowerment, Century Business, London. Clutterbuck D. (1998) 'Empowerment as mutuality of benefit', Human Resource Development International, 1(1).

Garvin D. A. (1993) ‘Building a Learning Organization', Harvard Business Review, JulyAugust.

Ghoshal S. and Bartlett C. (1998) The Individualized Corporation: a fundamentally new approach to management: great companies are defined by purpose, process, and people, Heinemann, London.

Harrison R. (1997) Employee Development, IPD, London.

Hicks R. F. and Bone D. (1991) Self-Managing Teams, Kogan Page, London. Megginson D., Joy-Matthews J. and Banfield P. (1993) Human Resource Development, IPD, London.

Mitchell Stewart A. (1994) Empowering People, Pitman, London.

Morton C. (1994) Becoming World Class, MacMillan, London.

O’Donnell D. and Garavan T. N. (1997) 'Linking training policy and practice to organizational goals', Journal of European and Industrial Training, 21 (9)

Pateman C. (1970) Participation and Democratic Theory, Cambridge University Press, Cambridge.

Pedler M., Burgoyne J. and Boydell T. (1997) The Learning Company, McGraw Hill, London. 
Pfeffer J. (1994) Competitive Advantage Through People, Harvard Business School Press, Boston Mass.

Semler R. (1993) Maverick! The success story behind the world's most unusual workplace, Century, London.

Wilkinson A. (1997) 'Empowerment: theory and practice', Personnel Review, 27(1). 